\title{
Protée
}

\section{Le lien. Sur une danse des Wayãpi (Amazonie)}

\section{Jean-Michel Beaudet}

Volume 29, numéro 2, 2001

Danse et altérité

URI : https://id.erudit.org/iderudit/030626ar

DOI : https://doi.org/10.7202/030626ar

Aller au sommaire du numéro

Éditeur(s)

Département des arts et lettres - Université du Québec à Chicoutimi

ISSN

0300-3523 (imprimé)

1708-2307 (numérique)

Découvrir la revue

Citer cet article

Beaudet, J.-M. (2001). Le lien. Sur une danse des Wayãpi (Amazonie). Protée, 29(2), 59-66. https://doi.org/10.7202/030626ar

\section{Résumé de l'article}

Ce texte propose une description et une analyse simple d'une danse amazonienne. Les Wayãpi, peuple du plateau des Guyanes, ne mettent en oeuvre que des danses collectives, où les danseurs sont en même temps instrumentistes et chanteurs. Dans cette danse en chaîne, où les interprètes se tiennent par l'épaule, le rythme semble se fonder sur le souffle commun plutôt que sur une pulsation préétablie. Enfin, ce souffle commun ne semble être possible, ne réussir que si les hommes, qui jouent des instruments à vent, prennent appui sur leur " cavalière " pour respirer. Ainsi, cette présentation tente de comprendre comment cette forme musicochorégraphique associe les différences (individuelles, de sexe, etc.), la posture respiratoire et le mouvement d'ensemble. 


\section{LE LIEN \\ SUR UNE DANSE DES W AYÃPI (AMAZONIE)}

JEAN - M ICHEL BEAUDET

Cette jeune fille appelle son danseur "papa» (en termes ethnologiques, il est le frère classificatoire de son père). C'est la première fois qu'elle participe à une danse; elle le fait de sa propre initiative. Je ne suis pas dans les confidences ou les bavardages de ces jeunes filles qui ont environ 16 ans; je sais simplement qu'elle est libre de danser ou non, de choisir celui avec qui elle danse, de se parer ou de se peindre le corps. Toutefois je puis dire que sa principale motivation est de se montrer, d'exposer la beauté de sa jeunesse. Cela est en soi un comportement exceptionnel, un comportement de fête, de danse. En effet, par l'éducation qu'elles reçoivent, les jeunes filles de ce village ont en général un comportement gestuel et spatial discret (leurs attitudes sonores peuvent l'être moins). Mais on voit qu'ici la grâce même du geste est faite de discrétion: aucune tension musculaire, pas de prise, le poignet est comme posé. C'est la bonne manière de tenir son danseur.

Ce lien de la main d'une femme et du bras de son danseur est à l'image de l'ensemble de l'esthétique chorégraphique wayãpi. C'est une disposition simple et qui n'est pas propre à cette culture, qui pour un regard extérieur ne serait pas marquante, pas particulière. Pourtant, au sein du monde wayãpi lui-même, c'est un geste hautement signifiant; il est exceptionnel, littéralement spectaculaire: jamais dans la vie quotidienne une jeune fille, une femme ne tient de cette manière le bras d'un homme quel qu'il soit (père, frère, mari, amant...). Racontant un épisode de la résistance aux envahisseurs brésiliens vers $1870^{1}$, Miso, grand connaisseur de l'Histoire de son peuple, précise - il répète même ce détail plusieurs fois: lorsque Asingau, le chef du village, invective les colons, leur dit de ne pas revenir, qu'il n'a pas peur d'eux malgré leurs crimes et leurs menaces, lorsqu'il leur dit ainsi, face à face, qu'il est prêt à les combattre et à se venger, sa femme est à ses côtés, lui tenant le bras.

Enfin, cette délicatesse de la prise, ce relâchement de la main, ce que j'ai résumé sous la valeur de discrétion, n'est pas facile à conserver lorsque l'ensemble de la chaîne des danseurs se déplace à des tempi variables. Et la difficulté à conserver tout au long de la danse les qualités plastiques de cette disposition la constitue comme véritable composante chorégraphique, comme participation jugée par les spectateurs, comme responsabilité ressentie par la danseuse. 


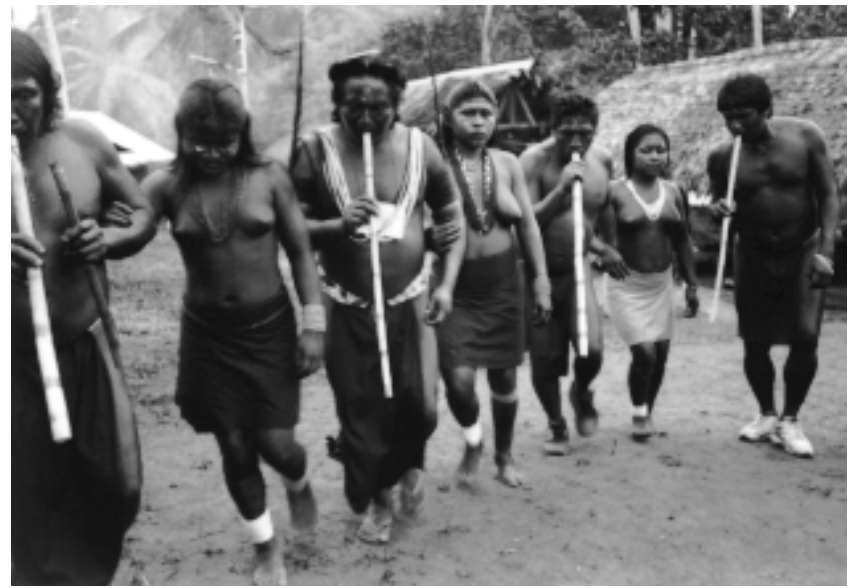

Figure 1

Danse yawalunã, 1995. Photo : Jean-Michel Miso.

LA FORME DE LA DANSE

Les deux photos ci-dessus sont extraites d'une prise de vue vidéo réalisée en novembre 1995 dans le village Zidock, sur le haut Oyapock, en Guyane française². Jacky Pawe, le premier danseur, est considéré par beaucoup comme le plus grand connaisseur des danses wayãpi de ce fleuve; en tout cas, c'est à lui que le plus souvent on demande d'organiser et de mener ces grandes danses. Jacky Pawe avait dit qu'il mènerait la danse yawalunã, "parce qu'elle n'a pas été dansée depuis longtemps... je veux que mon fils la connaisse». Elle n'a pas été dansée depuis au moins 1971, date où le père de Jacky est décédé. Le fils de Jacky, son seul garçon, est né en 1988 après cinq filles; aussi est-il particulièrement choyé par son père qui lui confectionne des couronnes de plumes, des arcs, des flûtes... On perçoit ici l'importance de la patrilinéarité dans la transmission des biens symboliques, et particulièrement des grandes danses.

"Yawalunã, ce sont deux animaux qui dansent ensemble, a dit Jacky, la martre eila et le jaguarundi yawalũ ${ }^{3}$. La plupart des grandes danses wayãpi sont consacrées à une espèce animale ou végétale - le poisson paku, le poisson suluwi, le palmier wasey, etc.-, ou à un ensemble générique - les oiseaux par exemple. J'ignore la raison pour laquelle ces deux petits carnivores se retrouvent ensemble dans cette danse, mais il est vraisemblable que cette bipolarité - c'est presque une danse bicéphale - soit exprimée ou produite par l'importance presque égale accordée aux deux sens de circulation de la chaine des danseurs. À la

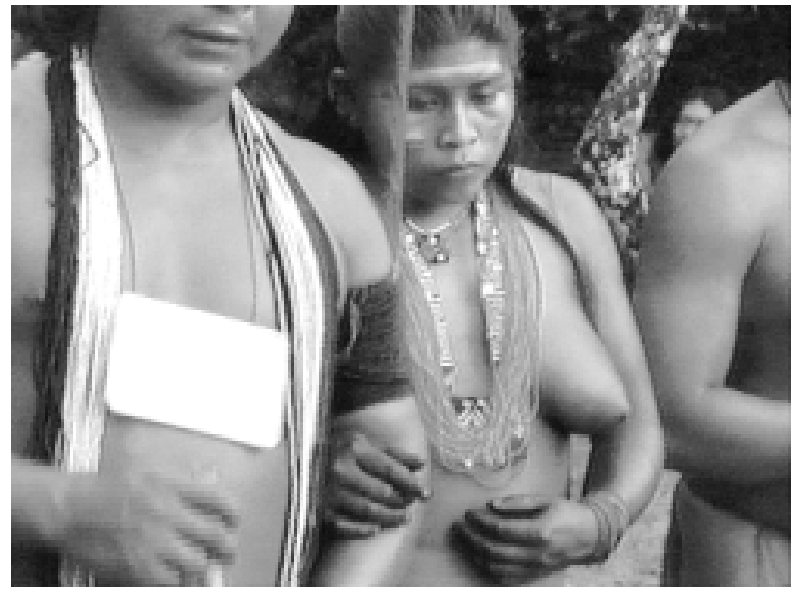

Figure 2

Danse yawalunã (détail).

différence du groupe des danses des oiseaux interprétées de préférence la nuit, yawalunã est exclusivement une danse de jour. Enfin, comme la presque totalité des autres grandes danses wayãpi, celleci se structure en une alternance de séquences chantées et de séquences instrumentales. Les hommes sont en même temps danseurs et chanteurs ou musiciens, ce qui est très fréquent en Amérique indigène.

Comme pour la majorité des musiques indigènes d'Amérique du Sud, on observe une correspondance forte entre formation instrumentale et répertoire. Les séquences instrumentales de yawalunã sont exécutées avec un seul type d'instruments: les ama'tatt, «bois Cecropia - nombreux", qui sont des clarinettes à trois anches, de 60 à $110 \mathrm{~cm}$ de long. Par ces deux caractéristiques associées - danse de jour, clarinette ama'tati -, cette danse est regroupée avec mãgãgã, la danse des bourdons, et eila, la danse de la martre. Cette répartition du répertoire en groupes de danses (une douzaine de danses des poissons, une douzaine de danses des oiseaux, trois danses des palmiers, etc.) a-t-elle une équivalence dans le jeu social (groupe de parenté, faction ou groupe local)? Est-ce que chaque village devrait avoir un chanteur capable de mener des danses des poissons, un autre spécialiste des danses des oiseaux, comme cela semble se dessiner dans certains grands villages? Chaque communauté villageoise, dramatiquement réduite par les épidémies du siècle passé, a-t-elle tendance à présenter son répertoire propre comme un emblème? 
Comme toutes les danses wayãpi, comme de nombreuses danses amazoniennes, celle-ci se présente d'abord comme un défilé : le dispositif de base est une chaîne de danseurs qui circule sur la place du village.

M'inspirant de Guilcher (1971), j'emploie «chaîne» pour une disposition où les danseurs se tiennent entre eux. Dans les danses des campagnes françaises qu'a décrites Guilcher, les danseurs se tiennent par la main, tandis que chez les Wayãpi, les danseurs se tiennent fondamentalement par l'épaule. Cette organisation chorégraphique de base présente des différences, synchroniques ou séquentielles: dans la majorité des séquences de toutes leurs danses, les hommes wayãpi tiennent l'épaule de celui ou celle qui précède (main droite - épaule gauche), tandis qu'une femme tient le bras gauche de celui qui la précède. Sur la photographie, on peut le voir, les hommes ne se tiennent pas; c'est une particularité des séquences instrumentales de cette danse yawalunã. Dans les autres danses wayãpi, les danseurs se tiennent presque en permanence par l'épaule. Pour cette danse yawalunã, ils ne le font que lors des séquences chantées, alors que les femmes ne lâchent jamais le bras de leur danseur. J'espère parvenir, grâce aux descriptions qui suivront, à faire sentir que ce lien est un élément syntaxique crucial de l'esthétique chorégraphique wayãpi et plus généralement, comme je l'ai dit ailleurs (1999), des esthétiques chorégraphiques des basses terres d'Amérique du Sud. Ainsi, le lien dans la danse serait à envisager comme une des techniques rituelles fondamentales de ces civilisations. On voit en tout cas que ce lien, caractéristique formelle de première importance, opère déjà une sexuation très forte de la danse. Une formulation inverse serait plus juste: notre jeune fille, en tenant ainsi le bras de son danseur, expose, outre sa beauté et sa discrétion, sa connaissance des formes chorégraphiques de son peuple et, se plaçant par sa participation à la danse dans le flux de la tradition, contribue à produire de la différenciation sexuelle.

Un autre élément de distinction entre les hommes et les femmes dans la chorégraphie se situe au niveau des mouvements mêmes de chaque danseur. Le mouvement de base de toute danse wayãpi est une marche balancée, pied à plat avec accentuation du pas droit. Là encore, ce boitement, peu spectaculaire en lui-même, est un type de pas exceptionnel pour les Wayãpi dont la démarche habituelle est celle des déplacements en forêt, démarche que l'on peut décrire sommairement comme légère et prudente. Ce mouvement fondamental de la danse est celui des hommes; les femmes ne marquent pas le pas. Pour tous et toutes, la tête, les épaules, les bras, les hanches ne bougent pas, le torse restant toujours d'une seule pièce. Cette fixité systématique est encore plus sensible pour les femmes qui, à la différence des hommes, restent toujours très droites. La dissymétrie du pas des hommes ainsi que la différenciation sexuelle qui s'opère à travers ce mouvement et cette posture de base sont largement répandues dans les basses terres d'Amérique du Sud.

On le voit donc, tous les hommes, d'une part, toutes les femmes, d'autre part, ont la même posture, font dans le même temps les mêmes mouvements: homorythmie et homokinésie. Ce dernier terme est un néologisme que je définis de la manière suivante: qualité qui caractérise l'action de deux personnes ${ }^{4}$ ou plus, qui s'appliquent à avoir la même posture, le même mouvement et le même tonus musculaire. L'homorythmie gestuelle désignerait donc un mouvement d'ensemble où plusieurs personnes pourraient faire des mouvements distincts mais coordonnés entre eux du point vue du temps. Dans l'homokinésie, les acteurs font des mouvements identiques, mais qui peuvent être non coordonnés du point de vue rythmique (décalés, comme dans un canon vocal, ou encore sur des pulsations différentes, etc.). L'addition de l'homorythmie et de l'homokinésie résulte en ce qu'on peut nommer un unisson gestuel ${ }^{5}$, ce qui est un des traits généraux des danses wayãpi.

On le voit aussi, la différenciation sexuelle est accentuée par l'homokinésie des genres. De plus, nous l'avons dit, les danseurs sont aussi, d'une séquence à l'autre, instrumentistes puis chanteurs. Lorsqu'ils chantent, les hommes produisent un unisson 
prononcé, où même le timbre des voix doit être neutralisé. En revanche, les femmes chantent les mêmes paroles, le même thème musical, au même tempo, mais en évitant d'être coordonnées entre elles et d'être coordonnées aux hommes pour ce qui est des hauteurs et du démarrage des phrases. On entend donc un mélange aigu, ce que j'ai appelé un «canon hétérogène» des femmes ${ }^{6}$, superposé à une homophonie grave des hommes. L'opposition coordination-incoordination est un axe différentiel fondamental pour de nombreuses formes musicales du monde ${ }^{7}$ et en particulier pour les musiques amérindiennes. Chez les Wayãpi, ce contraste présente différentes modalités de mise en œuvre selon les répertoires, et selon les séquences d'une même danse. Lors des séquences instrumentales de la danse yawalunã décrite ici, tous les musiciens soufflent en même temps sur la même pulsation qui est celle de leur pas de danse, produisant ainsi une base homorythmique relativement claire, sur laquelle pourra se développer l'ensemble des mouvements ${ }^{8}$.

On aura perçu aussi que dans la description des danses j'ai pris le parti de commencer par l'ensemble des danseurs et non par les mouvements individuels. En effet, là est un problème central du point de vue de l'analyse de la danse: l'être ensemble, le rythme général, la synchronisation. Tout cela, semble-t-il, est réalisé ici non par une addition d'individualités, mais à partir de la chaîne des danseurs, la chaîne étant ellemême le produit d'une collectivité plus grande, le village, qui participe à la danse par son regard, son attention, sa tension, sa prise en charge du risque global, et enfin par la programmation du service de la bière qui est effectué tout le long de la cérémonie. La synchronisation, la coordination rythmique des danseurs ne consiste pas ici à essayer de se placer sur une pulsation isochrone préétablie, mais plutôt à produire et démontrer une alliance entre le souffle et le pas ${ }^{9}$. Les ethnomusicologues commencent à remettre en cause l'usage a priori de la notion de pulsation isochrone (le découpage du flux temporel en unités de durées égales); de cet «impérialisme» du métronome venait sans doute la difficulté que j'avais à analyser la vitesse de ces danses, à dégager un tempo. Ici, il semblerait que la danse participe à la recherche d'un souffle commun. Dans cette danse, dans les conceptions wayãpi de la temporalité, le rythme, audelà d'une organisation du temps, serait une manière d'être ensemble ${ }^{10}$.

\section{ENSEMBLE}

Le rapport entre individu et groupe est un thème qui semble beaucoup intéresser les Wayãpi; ils le traitent en effet selon des modalités diverses et chaque fois longuement répétées, à travers toutes leurs formes musico-chorégraphiques. Dans les grandes danses ${ }^{11}$, la succession régulière des séquences instrumentales et des séquences chantées fait alterner tout au long de la journée différentes manières d'être ensemble, c'est-àdire des discours et des expériences différentes sur ce que peut être un ensemble d'individus.

Ces danses associent différents langages sensibles (gestuels, sonores, etc.) dont la plupart des signifiants ne sont pas arbitraires, mais évoquent directement un contenu, s'ajoutent pour faire apparaître les idées voisines d'ensemble d'individus, d'union, de coordination, voire même de discipline de groupe:

- chaque danseur exécute le même répertoire au même moment;

- ils sont habillés de manière semblable (parfois ils portent le même masque);

- ils se tiennent entre eux;

- ils ont la même posture, ils tiennent un autre danseur de la main droite et un instrument de la main gauche;

- il s'agit du même objet, du même instrument de musique;

- les gestes sont synchrones;

- leurs gestes ont la même forme, la même intensité; ils sont homogénéisés, neutres, c'est-à-dire moulés dans un style commun à toute la chaîne des danseurs;

- les hommes chantent en homophonie;

- ils chantent d'une voix neutre, tous avec la même intensité.

Toutefois, lorsqu'on considère d'un peu plus près ces différents paramètres, on voit que cette fusion, cette homogénéité n'est pas parfaite: les danseurs, 
quoique synchrones, ne le sont pas exactement; certains frappent un peu plus fort de leur pied droit, d'autres avec un peu de retard, d'autres encore s'arrêtent une fraction de seconde, ces petites différences individuelles étant aléatoires, non coordonnées. Ce n'est pas un défilé militaire, ni même une de ces danses d'Océanie où la synchronie est une caractéristique esthétique majeure (danse ma'ulu'ulu de Tonga, danse à figures kanak par exemple ${ }^{12}$ ). Autrement dit, il s'agit plus d'une intention démontrée d'homophonie et d'homokinésie que de synchronisation parfaite. La convergence des signifiants (danser ensemble, chanter ensemble, etc.) est accompagnée d'une réalisation de ces signifiants modérément contrainte; l'ajustement des mouvements du corps (voix comprise) n'est pas exact. Là semble être exprimée une deuxième idée importante: la personne individuelle n'est pas effacée; même lorsque les danseurs sont masqués, c'est-à-dire lorsqu'ils n'ont plus de nom, plus de position dans la parenté, il est accordé à chaque personnage dansant une part particulière d'expressivité. L'ensemble est fait d'individualités. Cette formulation pourrait laisser entendre qu'il s'agit d'un choix explicite. Bien au contraire, cette inexactitude est considérée dans le sens commun wayãpi comme «naturelle». L'exactitude et la perfection sont inconcevables; marcher au pas dans la forêt serait une aberration.

Fin de séquence instrumentale, les danseurs élargissent le cercle, tournent encore plusieurs fois dans les deux sens et enfin concluent cette séquence par une série de demi-voltes souples et puissantes. Ces tours-pivots ${ }^{13}$ constituent, avec les changements de sens de la chaîne des danseurs, une des caractéristiques principales de cette danse de la martre et du jaguarundi. Au tout début de la danse, c'est la seule indication que le meneur, Jacky, avait donnée aux autres danseurs: "Nous allons nous retourner». Le changement de sens de l'ensemble du dispositif est nommé oylowa, «ils se retournent», tandis que les
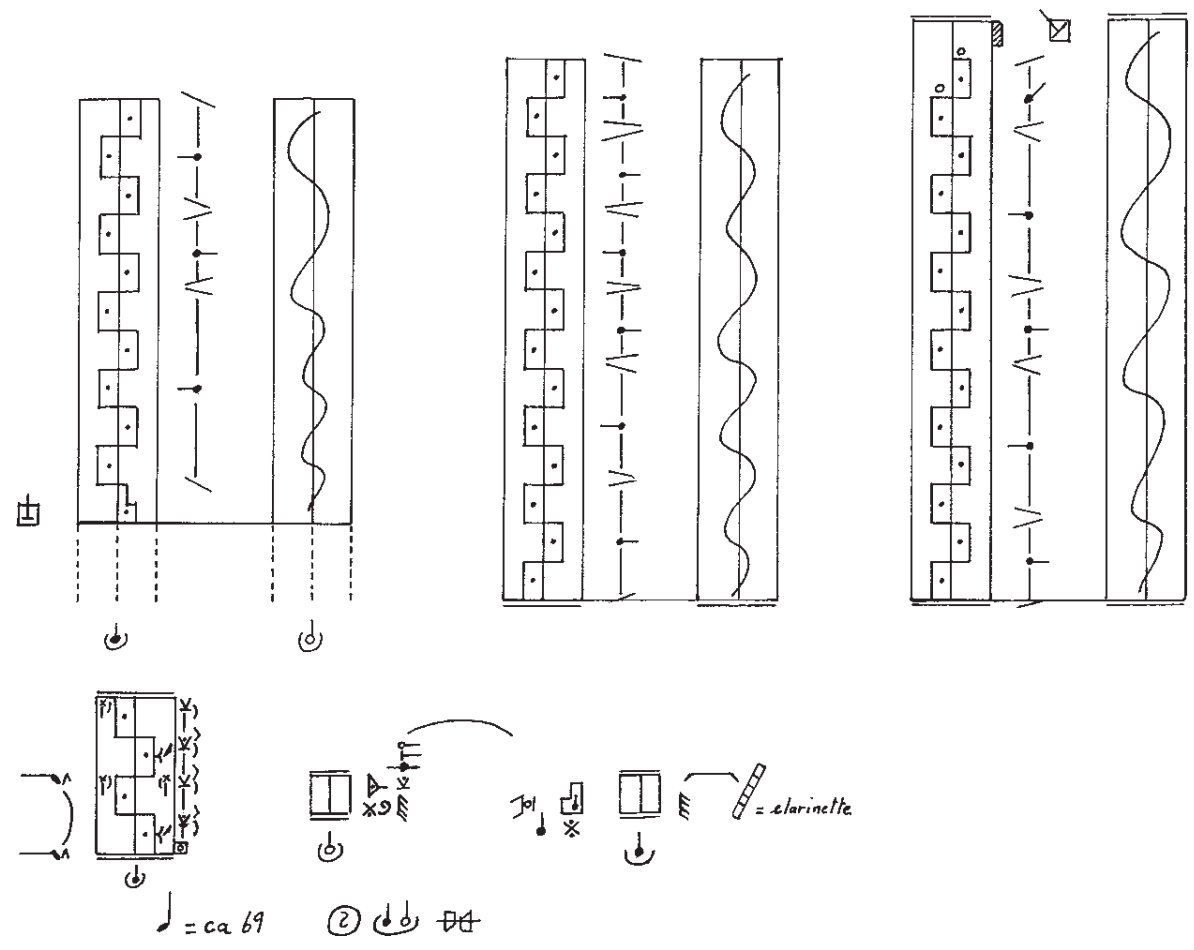

Figure 3

Fin de séquence instrumentale, série de 14 tours-pivots sur place par Jacky (transcription Laban). 

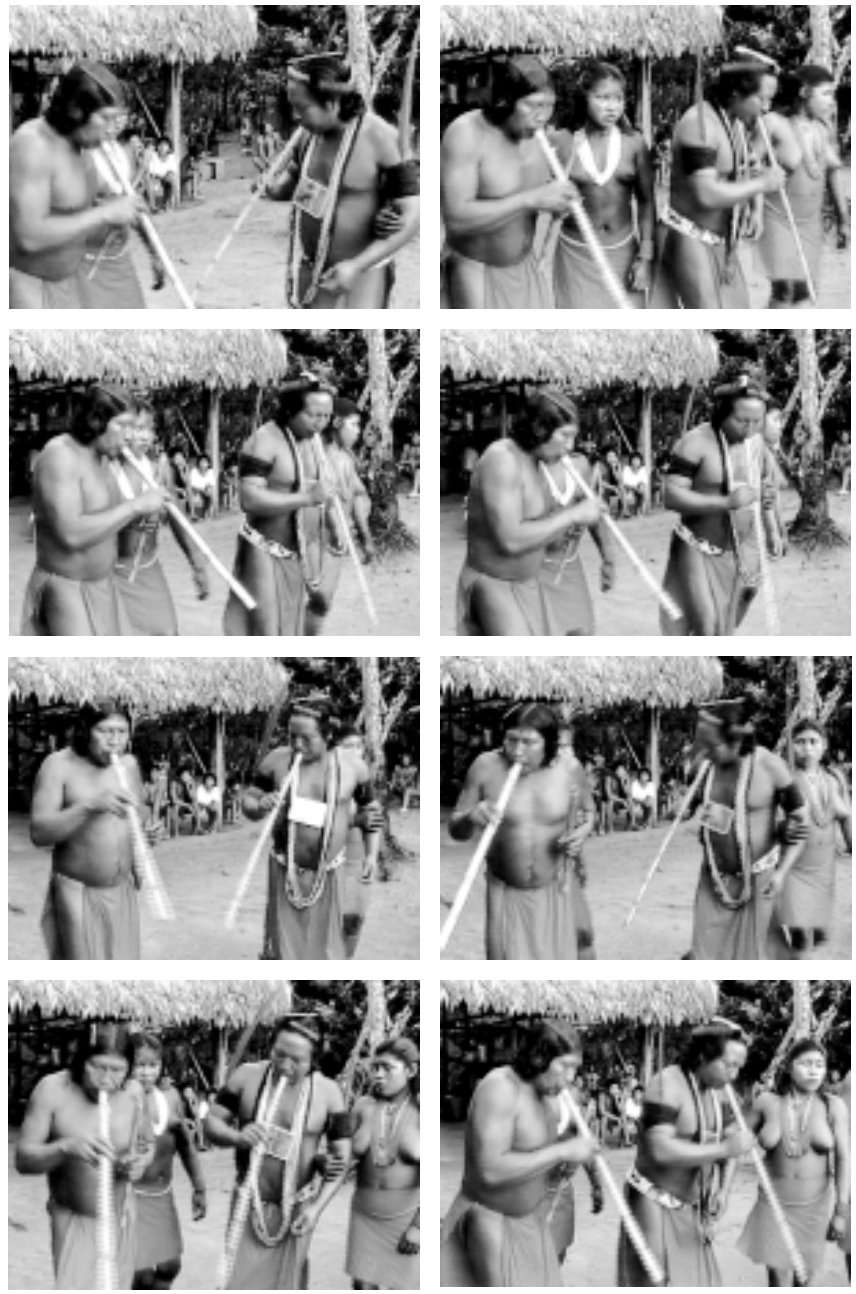

Figure 4

Fin de séquence instrumentale.

tours-pivots successifs sont nommés oylowalowa, «ils se tournent et se retournent» (-lowa «tourner la face vers", vient de la racine -owa, "visage»). Les tourspivots sur place sont réalisés à différents moments de chaque séquence, mais ils y occupent systématiquement une position syntaxique clé: ils ouvrent et ferment les séquences instrumentales, en constituent les formules introductives et conclusives. En début de séquence instrumentale, les tours-pivots sont moins nombreux et plus brefs: environ six retournements sur deux pas. Les tours-pivots s'effectuent selon un angle de $90^{\circ}$ à $140^{\circ}$ : ce ne sont pas, en général, des demi-voltes complètes, mais cet angle est suffisant pour exprimer le retournement, le changement d'orientation sur place.
Ces retournements se réalisent selon un nombre de pas variable, de deux à cinq, ainsi que selon des tempi et des durées variables. Angle, nombre de pas, tempo et durée varient à l'intérieur de chaque séquence de chaque danseur, selon son goût, ainsi que d'un danseur à l'autre. Lorsque le danseur de tête commence ses tours-pivots, les autres le suivent, mais les retournements des différents danseurs ne sont pas synchrones entre eux. On a bien, dans la chaîne de danse, une relative homorythmie de la musique et des pas; tout cela se fait à peu près sur la même pulsation, sans que les tempi des différents danseurs soient exactement identiques. Mais, surtout, les différents danseurs ne commencent pas les tours-pivots sur le même pas, certains d'entre eux les font sur deux pas tandis que d'autres les font sur trois pas, etc. Il est possible que cela soit une approximation, tolérée pour une danse qui n'avait pas été présentée depuis plus de vingt-cinq ans, mais je crois plutôt que ce désordre des retournements fait partie de l'esthétique même de yawalunã. Ce serait, là encore, une figuration des petits carnivores, thème de la danse. J'y vois aussi avec plaisir ce que le danseur contemporain Steve Paxton disait à propos de la "composition avec hasard »; cette danse affirmerait: nous sommes dans une civilisation, dans un moment, où l'esprit ne prend pas toutes les décisions.

\section{UNE RESPIRATION MIXTE?}

C'est très simple et évident pour tous, encore importe-t-il de ne pas l'oublier dans la compréhension d'une danse: que ce soit une danse de couple lié, une valse par exemple, de couple non lié, comme une cueca chilienne, ou une danse en chaîne d'un seul genre comme la chaîne xavante, ou encore une chaîne mixte avec alternance homme-femme comme un branle breton ou une grande danse wayãpi, dans toutes ces possibilités, après le mouvement de base, le deuxième grand niveau syntaxique de l'organisation du plaisir est celui du mouvement conjoint, c'est-àdire d'un mouvement où un(e) autre danseur(se) est associé(e) (ce que j'appelle les «niveaux syntaxiques d'organisation du plaisir" seraient au nombre de trois 
dans les chorégraphies wayãpi: l'ensemble de la chaîne, le couple homme-femme, le mouvement de base).

Que ressent-on (que ressent l'homme) dans ce mouvement conjoint wayãpi? Le plaisir d'une présence autre, bien sûr, une suite légère, comme une aile gauche qui en même temps battrait de manière assez indépendante en suivant et en amplifiant les rotations, reculs, changements de direction du danseur... et qui en même temps donc transmettrait ces mouvements aux danseurs suivants. Il en résulte que les mouvements des danseurs de la chaine sont à la fois synchrones et successifs: ils sont sentis et vus à la fois comme une simultanéité et comme une onde qui se propage (une onde qui se propage de manière peu régulière) ${ }^{14}$.

Reprenons les différences liées au sexe des acteurs dans les danses wayãpi. Les danseuses entrent toujours dans la danse après les hommes. Elles se glissent au bras de leur danseur à la troisième ou quatrième strophe, soit une bonne heure après le début de la danse. De plus, elles quittent plusieurs fois la chaîne au cours de l'ensemble de la chorégraphie, alors que tous les hommes y demeurent, présents et actifs, du début à la fin. Cette distinction est répandue dans les basses terres d'Amérique du Sud et, à travers elle, les hommes se réaffirment notamment comme les responsables de la danse.

Pour toute danse wayãpi - les femmes tenant le bras gauche de leur danseur, et l'ensemble tournant dans le sens anti-horaire -, les femmes sont à l'intérieur du cercle. La chorégraphie de yawalunã a pour particularité, nous l'avons vu, de présenter des changements systématiques de sens de circulation de la chaîne, faisant que celle-ci avance presque à part égale dans le sens horaire. Alors les femmes se placent à la droite de leur danseur, confirmant ainsi cette loi générale de la culture wayãpi, loi qui semble assez répandue en Amazonie: les femmes dansent à l'intérieur du cercle, les hommes sont à la périphérie ${ }^{15}$.

$\mathrm{Si}$, de manière générale dans l'esthétique chorégraphique wayãpi, le pas est dissymétrique ${ }^{16}$, on voit que le lien est lui aussi dissymétrique (main de la femme - bras de l'homme/main de l'homme - épaule de la femme).

Enfin, au niveau du mouvement de base, le fait que les hommes boitent de manière accentuée, tandis que les femmes ne marquent pas le pas, se traduit en termes de flux et d'action ${ }^{17}$ par un contraste entre des mouvements continus chez les femmes, discontinus chez les hommes. Visuellement, pour le spectateur, ce contraste donne aux femmes une apparence de légèreté ${ }^{18}$.

Il ne m'est pas possible encore de donner une interprétation générale de ces distinctions de genre dans les danses wayãpi. Néanmoins, on peut résumer cet ensemble de contrastes en observant qu'ainsi, sur cette "scène", les femmes ne jouent pas de rôle; elles ne portent pas, ne se donnent pas de masques. $\mathrm{Ni}$ capes d'écorces que se confectionnent les hommes pour certaines performances, ni musique instrumentale, ni postures ou mouvements des animaux représentés - chacun de ces trois langages sensibles pouvant en effet être interprété comme un masque. Elles ne se transforment pas, elles demeurent des femmes wayãpi dans leur village, au bras de leurs hommes, hommes triplement masqués, triplement dans le risque de la transfiguration. Dans la vie non dansée, les femmes se transforment peu; elles sont beaucoup moins que les hommes en contact avec les mondes étrangers - la chasse, le chamanisme, la guerre, le troc étant des formes d'échange qui s'associent, pour les Wayãpi, à des pratiques masculines. Les hommes frappent le sol, s'appuient dessus, les femmes, elles, apparaissent comme légères. Alors que danser hors du village est inconcevable, ce ne sont pas les humaines qui dessinent l'espace de danse, se sont les masqués, les transfigurés qui marquent ce territoire. Mais la posture du couple dans la danse pose une autre question importante de cette étude: quel est le lien entre souffle, rythme et mouvement du corps ${ }^{19}$ ? Nous l'avons vu, les hommes wayãpi dansant soufflent dans des aérophones et, pour les grandes danses, cela est fort éprouvant, cela demande de grandes capacités respiratoires, et surtout du rythme. Or, il semblerait que ce rythme, cette 
aisance respiratoire soit donnée par les danseuses: le danseur inspire à chaque pulsation, à chaque pas, tout en levant le buste, en tirant un peu la main, le lien de sa danseuse. Sa respiration "prend appui» sur le nonmouvement de sa danseuse. Si l'homme ne se laisse pas tenir par la légèreté de la femme, il ne peut pas respirer. Main droite de la femme sur le bras gauche de l'homme, bras gauche qui lui-même tient un aérophone, danseur qui souffle dans cet instrument tout en tenant de la main droite l'épaule de la danseuse qui le précède - cet ensemble constitue un maillon complet de la chaîne. Lorsque la compréhension motrice de ce lien entre l'homme et sa danseuse est trouvée, alors la danse trouve son rythme, sa beauté.

\section{N O TES}

1. C'est l'histoire de Sa'i Paye, «la grand-mère chamane»; elle réussit, sur la rivière Kouc, à repousser les colons brésiliens qui avaient détruit les plantations de manioc, assassiné le frère du chef Asingau, et faisaient pression de manière constante pour occuper son village. À la demande d'Asingau, «elle fit attaquer les canots des Brésiliens par des piranhas".

2. Les Wayãpi sont un peuple de langue et de culture tupi, vivant de part et d'autre de la frontière entre le Brésil et la Guyane française. Ils sont un peu plus d'un millier aujourd'hui (voir Albert, Menget et Razon, 1985; Gallois, 1999).

3. Eila: Eira barbara L.

Yawalũ: Herpailurus yaguaroundi Ca. et Ye.

4. Ou des animaux, ou des êtres vivants.

5. Je n'ose pas proposer le terme «d'unikinésie». Si le terme d'unisson bénéficie d'une définition à peu près consensuelle auprès des musicologues ou des ethnomusicologues, il n'en est pas de même de l'homophonie dont les définitions, malgré les efforts d'Arom (1985), ne parviennent pas à se dégager d'une confusion avec l'homorythmie. 6. Beaudet, 1997.

7. Un groupe de travail "polymusique-hétérophonie», constitué au sein du Laboratoire d'Ethnomusicologie du CNRS, tente actuellement d'élaborer des lignes d'analyse de ces formes musicales.

8. L'hétérogénéité sonore, prisée en général dans les séquences instrumentales des grandes danses, est ici «localisée » dans la multiplicité des anches, les anches - ni dans une seule clarinette, ni entre deux clarinettes - n'étant pas "accordées" entre elles. Le résultat est donc une succession régulière, une pulsation de clusters, chacun de ces clusters étant lui-même formé d'une addition simultanée de quinze "petits» clusters.

9. Voir Beaudet, 1997.
10. Je ne prétends en aucune manière faire de cette proposition une généralité: j'ai entendu, en Amazonie même, des musiques au tempo très ferme.

11. Les formes musico-chorégraphiques wayãpi peuvent être schématiquement réparties en deux répertoires: ce que je nomme «les grandes danses " se distinguent, par de nombreux traits, des suites pour ensembles de grandes clarinettes en bambou tule (cf. Beaudet, 1997). 12. Autre exemple, plus proche des Wayãpi, les danseurs xavante (peuple du Brésil central, de langue et de culture gé) produisent et s'imprègnent d'une synchronie puissante et très exacte. Une association entre une grande tension musculaire, une concentration sur soi (le regard est oblique, dirigé devant vers le sol), et une fluidité de l'ensemble: comme dans un chœur, la multiplication du même geste produit une sensation d'amplification de sa propre expression.

13. Oủ les hommes, accompagnés de leurs danseuses, procèdent à des séries de retournements, des demi-voltes, qu'on peut aussi appeler des tours-pivots pour employer le vocabulaire de la cinétographie Laban. 14. Dans la danse xavante mixte, ce que l'on ressent est la surprise produite par l'association entre, d'une part, le déplacement latéral du centre de gravité associé à un tronc d'une seule pièce de l'homme et, d'autre part, le pli, la cassure en avant de la femme qui balance ainsi les centres de gravité selon un mouvement pendulaire saccadé dans le plan sagittal.

15. Il y a bien sûr des exceptions remarquables mais pour lesquelles je ne puis encore donner d'interprétation: dans les danses des poissons wayãpi, les femmes des deux "danseurs-piranhas" sont toujours à l'extérieur.

16. Dissymétrique et non "asymétrique»: il me semble utile en effet de pouvoir grâce à ces deux termes maintenir une distinction analytique entre, d'une part, une absence totale de symétrie, l'asymétrie pouvant par exemple être associée à une signification de désordre, et, d'autre part, la dissymétrie qui, comme symétrie faussée, peut faire référence à une symétrie virtuelle - le boitement de la danse wayãpi est un bon exemple de dissymétrie des appuis, c'est-à-dire ici d'inégalité régulière dans le transfert du poids du corps.

17. Flux et action, termes proposés par Laban et dont l'ambiguité fut largement commentée et mise et perspective par H. Godard (séminaire doctoral du Département Danse, Université de Paris 8, 1999).

18. Beaudet, 1999.

19. Voir Godard, 1994.

\section{RÉFÉREN CES BIBLIO G RAPHIQ UES}

Albert, B., P. MEnget et J. RAZON (dir.) [1985]: «La question amérindienne en Guyane française ", Ethnies, 1-2, Paris, Survival International France.

AROM, S. [1985]: Polyphonies et polyrythmies instrumentales d'Afrique Centrale, Paris, Selaf.

BEAUDET, J.-M. [1997]: Souffles d'Amazonie, Nanterre, Société d'ethnologie;

[1999]: «Polay, uwa: danser chez les Wayãpi et les Kalina. Notes pour une ethnographie des danses amérindiennes des basses terres d'Amérique du Sud ", Journal de la Société des Américanistes, 85, 215-237.

GALLOIS, D. (dir.) [1999]: Terra Indigena Waiãpi : alternativa para o desenvolvimento sustentável, Belem, Centro de Trabalho Indigenista. GODARD, H. [1994] : «Le souffle, le lien », Marysas, 32, 27-31. GUILCHER, J.-M. [1971]: «Aspects et problèmes de la danse populaire traditionnelle», Ethnologie française, vol. I, nº 2, 7-48. 